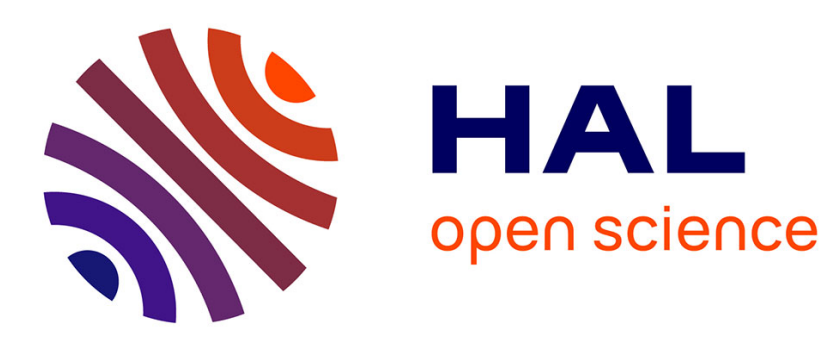

\title{
Direct and adjoint global modes of a recirculation bubble: Lift-up and convective non-normalities
}

\author{
O. Marquet, M. Lombardi, Jean-Marc Chomaz, D. Sipp, L. Jacquin
}

\section{To cite this version:}

O. Marquet, M. Lombardi, Jean-Marc Chomaz, D. Sipp, L. Jacquin. Direct and adjoint global modes of a recirculation bubble: Lift-up and convective non-normalities. Journal of Fluid Mechanics, 2009, 622 (march), pp.1-21. 10.1017/s0022112008004023 . hal-01021132

HAL Id: hal-01021132

https://hal-polytechnique.archives-ouvertes.fr/hal-01021132

Submitted on 16 Jul 2014

HAL is a multi-disciplinary open access archive for the deposit and dissemination of scientific research documents, whether they are published or not. The documents may come from teaching and research institutions in France or abroad, or from public or private research centers.
L'archive ouverte pluridisciplinaire HAL, est destinée au dépôt et à la diffusion de documents scientifiques de niveau recherche, publiés ou non, émanant des établissements d'enseignement et de recherche français ou étrangers, des laboratoires publics ou privés. 


\title{
Direct and adjoint global modes of a recirculation bubble: lift-up and convective non-normalities
}

\author{
OLIVIER MARQUET ${ }^{1}$, MATTEO LOMBARDI ${ }^{1}$, \\ JEAN-MARC CHOMAZ ${ }^{1,2}$, DENIS SIPP ${ }^{1}$ \\ AND LAURENT JACQUIN ${ }^{1}$
}

\author{
${ }^{1}$ Departement d'Aerodynamique Fondamentale et Experimentale, ONERA, 92190 Meudon, France \\ ${ }^{2}$ Laboratoire d'Hydrodynamique (LadHyx), CNRS - Ecole Polytechnique, 91128 Palaiseau, France
}

(Received 29 April 2007 and in revised form 20 July 2008)

The stability of the recirculation bubble behind a smoothed backward-facing step is numerically computed. Destabilization occurs first through a stationary threedimensional mode. Analysis of the direct global mode shows that the instability corresponds to a deformation of the recirculation bubble in which streamwise vortices induce low- and high-speed streaks as in the classical lift-up mechanism. Formulation of the adjoint problem and computation of the adjoint global mode show that both the lift-up mechanism associated with the transport of the base flow by the perturbation and the convective non-normality associated with the transport of the perturbation by the base flow explain the properties of the flow. The lift-up non-normality differentiates the direct and adjoint modes by their component: the direct is dominated by the streamwise component and the adjoint by the crossstream component. The convective non-normality results in a different localization of the direct and adjoint global modes, respectively downstream and upstream. The implications of these properties for the control problem are considered. Passive control, to be most efficient, should modify the flow inside the recirculation bubble where direct and adjoint global modes overlap, whereas active control, by for example blowing and suction at the wall, should be placed just upstream of the separation point where the pressure of the adjoint global mode is maximum.

\section{Introduction}

For many years the problem of transition to turbulence in shear flows has been addressed by studying the exponential growth of linear perturbations developing on a base flow. However this linear modal analysis failed to predict the transitional Reynolds number determined experimentally in parallel shear flows such as Couette and Poiseuille flows since those flows are linearly stable at this transitional Reynolds number. In the past decade a transition scenario based on the concept of receptivity has been considered that completes the modal analysis. The flow receptivity defines the ability of stable flows to amplify perturbation energy in a transient manner. This non-modal amplification is due to the non-normality of the linearized Navier-Stokes operator (Trefethen et al. 1993). The non-normality of parallel shear flows was first explored by Butler \& Farrell (1992) who described the physical mechanism underlying the non-normality of parallel shear flows as the so-called lift-up mechanism 
(Landahl 1980) which creates large streamwise perturbations through transport of the base-flow momentum by cross-stream velocity perturbations. Transition to turbulence is then due (see the review by Schmid 2007) to a nonlinear feedback between streamwise streaks and streamwise vortices and only a nonlinear weak feedback is sufficient since the linear non-normal amplification of vortices into streaks is extremely large and increases with the Reynolds number.

Open flows are usually strongly non-parallel and analysing their stability requires, in the case of a flow homogeneous in the spanwise direction, solving a two-dimensional eigenvalue problem for which both the streamwise and cross-stream directions are eigendirections. Thanks to increasing computational capacity, the so-called global stability analysis has recently received substantial attention. Theofilis, Hein \& Dallman (2000), Barkley, Gomes \& Henderson (2002), Gallaire, Marquillie \& Ehrenstein (2007) - among many others - have determined the spectrum of the linearized Navier-Stokes operator for different two-dimensional base flows. Only a few studies (Schmid \& Henningson 2002; Ehrenstein \& Gallaire 2005; Giannetti \& Luchini 2007; Akervik et al. 2007) have started to analyse the dynamics resulting from the non-normality of this operator. Most of them have focused on the description of the amplifier dynamics, i.e. the short-time dynamics resulting from a transient amplification of the perturbation energy. As shown by Schmid \& Henningson 2002 or Ehrenstein \& Gallaire (2005), this transient growth may be viewed as the superposition of global modes since they are non-orthogonal when the operator is non-normal. As suggested by Chomaz (2005), examining the non-normality of the evolution operator is also of interest to better understand the destabilization of the flow close to the bifurcation. To our knowledge, only Giannetti \& Luchini $(2003,2007)$ have perfomed such an analysis, in the case of a circular cylinder flow. They have computed both the direct and adjoint leading global modes that describe the occurrence of the von Kármán street and commented, as did Chomaz Huerre \& Redekopp (1990) and Cossu $\&$ Chomaz (1997) when studying the Ginzburg-Landau envelop equation with varying coefficients, on the dissymmetry due to the transport of perturbations by the base flow.

The objective of the present paper is to generalize this consideration to the threedimensional destabilization of a two-dimensional flow and to apply it to a recirculation bubble in S-shaped geometry modelling an air intake. In other configurations, Theofilis et al. (2000) for a flow separating on a flat plate, Barkley et al. (2002) for a backwardfacing-step flow and Gallaire et al. (2007) for separating flow over a bump, have found that the first destabilization, on increasing the Reynolds parameter, is threedimensional and steady. By computing both the direct global mode associated with the leading eigenvalue of the linearized Navier-Stokes operator and the adjoint global mode solution of the corresponding adjoint problem we discuss the non-normality of the flow and point out the interplay between the lift-up mechanism and the convective non-normality due to the transport of the perturbation by the base flow. For a study of the non-normality and the resulting amplifier dynamics for the present recirculation flow, we refer to Marquet et al. (2008).

The paper is organized as follows. In $\S 2$ we formulate the problem of the direct and adjoint global mode analysis, distinguish two origins of the non-normality by examining the direct and adjoint operators and thereby we extend the consideration developed in the review by Chomaz (2005). In $\S 3$ we present the numerical methods and convergence tests. In $\S 4$ we present the results of the direct and adjoint global stability analysis. In particular we discuss the impact of the two distinct origins of the non-normality pointed out in $\S 2$ on the direct and adjoint leading global 

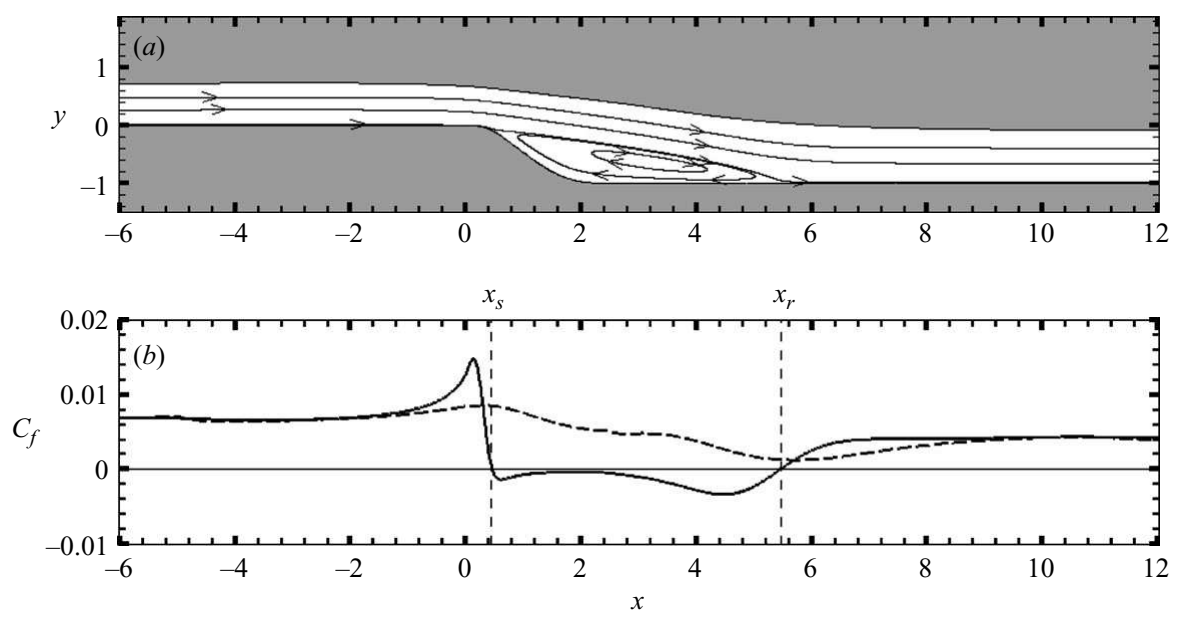

FIGURE 1. Flow configuration and base-flow characterization. (a) Streamlines of the base flow showing the recirculation region. (b) Skin-friction coefficient on the lower wall (solid curve) and upper wall (dashed curve) as a function of the non-dimensional longitudinal coordinate $x$; the separation $\left(x_{s}=0.46\right)$ and reattachment $\left(x_{r}=0.55\right)$ points defining the length of the recirculation bubble are shown. Parameter settings: $R e=800$, computational mesh $M_{b 1}$.

modes. Finally, we give a physical interpretation of the adjoint global mode in terms of receptivity of the global mode to initial conditions and forcing, as well as its sensitivity to local modification of the recirculation bubble, both properties being crucial when control is concerned.

\section{Problem formulation}

\subsection{Geometry and base flow}

A recirculation bubble is a separated flow reattaching downstream of the separation point, thus forming a recirculation region. To obtain such a flow configuration an S-shaped geometry (figure 1) has been designed. The shape of the lower wall is a rounded backward-facing step, defined by its height $h$ and its length $L$, and the shape of the upper wall has been chosen to prevent any recirculation region on this wall. Further details about the geometry are provided in Appendix A. Coordinates $x, y$ and $z$ denote respectively the longitudinal, vertical and transverse directions with origin taken at the rounded-step edge. Those Cartesian coordinate are made non-dimensional with the step height $h$. The computational domain is denoted $\Omega$ and delimited by the boundaries $\Gamma_{i}, \Gamma_{o}, \Gamma_{u}, \Gamma_{l}$ which correspond respectively to the inlet, the outlet and the upper and lower solid walls.

The motion of a viscous fluid governed by the non-dimensional incompressible Navier-Stokes equations is considered:

$$
\begin{aligned}
\partial_{t} \boldsymbol{u}+(\boldsymbol{u} \cdot \nabla) \boldsymbol{u} & =-\nabla p+R e^{-1} \nabla^{2} \boldsymbol{u}, \\
\nabla \cdot \boldsymbol{u} & =0,
\end{aligned}
$$

where $\boldsymbol{u}=(u, v, w)$ is the non-dimensional velocity field, $p$ is the pressure field scaled by $\rho U_{0}^{2}$ and the Reynolds number is defined as $R e=U_{0} h / v ; v$ is the kinematic viscosity of the fluid and $U_{0}$ is the maximum inlet centreline velocity which has been 
used to scale the velocities. The following boundary conditions are imposed:

$$
\begin{gathered}
u=\frac{4 y\left(h_{i}-y\right)}{h_{i}^{2}}, v=w=0 \text { on } \Gamma_{i}, \\
u=v=w=0 \text { on } \Gamma_{u} \cup \Gamma_{l}, \\
p-R e^{-1} \partial_{x} u=0, \partial_{x} v=\partial_{x} w=0 \text { on } \Gamma_{o} .
\end{gathered}
$$

They correspond respectively to a parabolic velocity profile at the inlet, no-slip conditions on the solid walls and a free outflow condition at the outlet. The coefficient $h_{i}$ in the definition of the parabolic profile is the inlet height.

\subsection{Base-flow computation and global modes analysis}

The velocity and pressure fields are decomposed into a two-dimensional base flow $\boldsymbol{Q}(x, y)=(\boldsymbol{U}, P)^{T}=(U, V, 0, P)^{T}$ and a three-dimensional disturbance flow, denoted by the quadrivector $\epsilon \boldsymbol{q}^{\prime}(x, y, z, t)=\left(u^{\prime}, v^{\prime}, w^{\prime}, p^{\prime}\right)^{T}$, where $\epsilon$ is the amplitude of the perturbation. The base flow is assumed to be a solution of the stationary twodimensional incompressible Navier-Stokes equations

$$
\begin{aligned}
(\boldsymbol{U} \cdot \nabla) \boldsymbol{U} & =-\nabla P+R e^{-1} \nabla^{2} \boldsymbol{U}, \\
\nabla \cdot \boldsymbol{U} & =0,
\end{aligned}
$$

associated with the boundary conditions $(2.2 a-c)$. At leading order in $\epsilon$ the perturbation $\boldsymbol{q}^{\prime}$ is a solution of the linearized Navier-Stokes equations and since the base flow is stationary and homogeneous in the transverse direction $z$, the threedimensional perturbations may be sought as a normal mode

$$
\boldsymbol{q}^{\prime}(x, y, z, t)=\frac{1}{2}\left\{(\hat{u}, \hat{v}, \hat{w}, \hat{p})^{T}(x, y) \exp [\mathrm{i} k z+\sigma t]+\text { c.c. }\right\},
$$

where $k$ is the real spanwise wavenumber, $\sigma$ the complex growth rate and c.c. stands for the complex conjugate of the preceding expression. The imaginary part $\sigma_{i}$ of the global mode growth rate $\sigma$ is related to the frequency of the mode, and its real part $\sigma_{r}$ to the temporal growth rate. In particular, $\sigma_{r}>0$ denotes exponential growth of the mode $\boldsymbol{q}^{\prime}$ in time $t$.

Use of this normal mode decomposition in the Navier-Stokes equation linearized around the base flow $\boldsymbol{Q}$ gives that the growth rate is solution of the generalized eigenvalue problem that may be written in the form:

$$
\boldsymbol{A} \cdot \hat{\boldsymbol{q}}=\sigma \boldsymbol{B} \cdot \hat{\boldsymbol{q}},
$$

where $\hat{\boldsymbol{q}}=(\hat{\boldsymbol{u}}, \hat{p})^{T}=(\hat{u}, \hat{v}, \mathrm{i} \hat{w}, \hat{p})^{T}$ is the complex eigenvector, the so-called global mode, associated with the complex eigenvalue $\sigma$, the global growth rate. As noticed by Theofilis (2000), the particular form of $\hat{\boldsymbol{q}}$ with the $\hat{w}$ component in quadrature with the three others allows the solution of a generalized eigenvalue problem (2.5) with real matrices $\boldsymbol{A}$ and $\boldsymbol{B}$ defined as:

$$
\boldsymbol{A}=\left(\begin{array}{cccc}
\mathrm{D}-\mathrm{C}-\partial_{x} U & -\partial_{y} U & 0 & -\partial_{x} \\
-\partial_{x} V & \mathrm{D}-\mathrm{C}-\partial_{y} V & 0 & -\partial_{y} \\
0 & 0 & \mathrm{D}-\mathrm{C} & k \\
\partial_{x} & \partial_{y} & k & 0
\end{array}\right) ; \quad \boldsymbol{B}=\left(\begin{array}{cccc}
1 & 0 & 0 & 0 \\
0 & 1 & 0 & 0 \\
0 & 0 & 1 & 0 \\
0 & 0 & 0 & 0
\end{array}\right)
$$

where $\mathrm{D}=R e^{-1}\left(\partial_{x^{2}}+\partial_{y^{2}}-k^{2}\right)$ accounts for the viscous diffusion of the perturbation and $\mathrm{C}=U \partial_{x}+V \partial_{y}$ for its advection by the base flow. 
The boundary conditions associated with (2.5) are

$$
\begin{gathered}
(\hat{u}, \hat{v}, \mathrm{i} \hat{w})=(0,0,0) \text { on } \Gamma_{u} \cup \Gamma_{l} \cup \Gamma_{i}, \\
\hat{p}-R e^{-1} \partial_{x} \hat{u}=0, \partial_{x} \hat{v}=\partial_{x} \hat{w}=0 \text { on } \Gamma_{o} .
\end{gathered}
$$

To simplify the discussion the eigenmodes, solutions of (2.5) and (2.7), will be assumed discrete and non-degenerate. They will be indexed by an integer $l$ and ranked in decreasing order of the real part of their temporal growth rate.

\subsection{Non-normality and adjoint analysis}

We define $\boldsymbol{A}^{+}$, the adjoint operator of $\boldsymbol{A}$, as the operator such that, for any vectors $\hat{\boldsymbol{q}}$ fulfilling the boundary conditions (2.7) and $\hat{\boldsymbol{q}}^{+}$, with respect to boundary conditions to be determined,

$$
\left(\hat{\boldsymbol{q}}^{+}, \boldsymbol{A} \cdot \hat{\boldsymbol{q}}\right)=\left(\boldsymbol{A}^{+} \cdot \hat{\boldsymbol{q}}^{+}, \hat{\boldsymbol{q}}\right)
$$

where $\hat{\boldsymbol{q}}^{+}=\left(\hat{\boldsymbol{u}}^{+}, \hat{p}^{+}\right)^{T}=\left(\hat{u}^{+}, \hat{v}^{+}, \mathrm{i} \hat{w}^{+}, \hat{p}^{+}\right)^{T}$ and $(.,$.$) corresponds to the inner product$

$$
\left(\hat{\boldsymbol{q}}^{+}, \hat{\boldsymbol{q}}\right)=\int_{\Omega} \hat{\boldsymbol{q}}^{+t} \cdot \hat{\boldsymbol{q}} \mathrm{d} \Omega .
$$

The superscript $t$ denotes the transconjugate and the centred dot refers to the usual scalar product of two vectors. If the instantaneous energy density of a threedimensional perturbation is defined as

$$
E\left(\boldsymbol{q}^{\prime}\right)=\frac{1}{L_{z} \Omega} \int_{-L_{z} / 2}^{+L_{z} / 2} \int_{\Omega}\left(u^{\prime 2}+v^{\prime 2}+w^{\prime 2}\right) \mathrm{d} x \mathrm{~d} y \mathrm{~d} z,
$$

where $L_{z}=2 \pi / k$ is the transverse wavelength, $\Omega$ being the computational domain previously defined, it can be expressed using the normal mode decomposition (2.4) as

with

$$
E\left(\boldsymbol{q}^{\prime}\right)=\frac{1}{2} E(\hat{\boldsymbol{q}}) \exp \left[2 \sigma_{r} t\right]
$$

$$
E(\hat{\boldsymbol{q}})=(\hat{\boldsymbol{q}}, \boldsymbol{B} \cdot \hat{\boldsymbol{q}}) .
$$

By successively integrating the left-hand side of (2.8) by parts, we obtain

$$
\boldsymbol{A}^{+}=\left(\begin{array}{cccc}
\mathrm{D}+\mathrm{C}-\partial_{x} U & -\partial_{x} V & 0 & -\partial_{x} \\
-\partial_{y} U & \mathrm{D}+\mathrm{C}-\partial_{y} V & 0 & -\partial_{y} \\
0 & 0 & \mathrm{D}+\mathrm{C} & k \\
\partial_{x} & \partial_{y} & k & 0
\end{array}\right),
$$

and the adjoint boundary conditions:

$$
\left.\begin{array}{rl}
\left(\hat{u}^{+},\right. & \left.\hat{v}^{+}, i \hat{w}^{+}\right)=(0,0,0) \text { on } \Gamma_{u} \cup \Gamma_{l} \cup \Gamma_{i}, \\
& \hat{p}^{+}-R e^{-1} \partial_{x} \hat{u}^{+}=-\hat{u}^{+} U \\
& R e^{-1} \partial_{x} \hat{v}^{+}=\hat{v}^{+} U \\
& R e^{-1} \partial_{x} \hat{w}^{+}=\hat{w}^{+} U
\end{array}\right\} \text { on } \Gamma_{o} .
$$

appropriate to cancel all of the boundary terms appearing during the integration by parts. The adjoint eigenproblem is then

$$
\boldsymbol{A}^{+} \cdot \hat{\boldsymbol{q}}^{+}=\sigma^{+} \boldsymbol{B} \cdot \hat{\boldsymbol{q}}^{+},
$$

$\sigma^{+}$and $\hat{\boldsymbol{q}}^{+}$being the adjoint growth rate and the adjoint global mode. It may be shown (see Schmid \& Henningson 2001) that all the adjoint complex eigenvalues, 

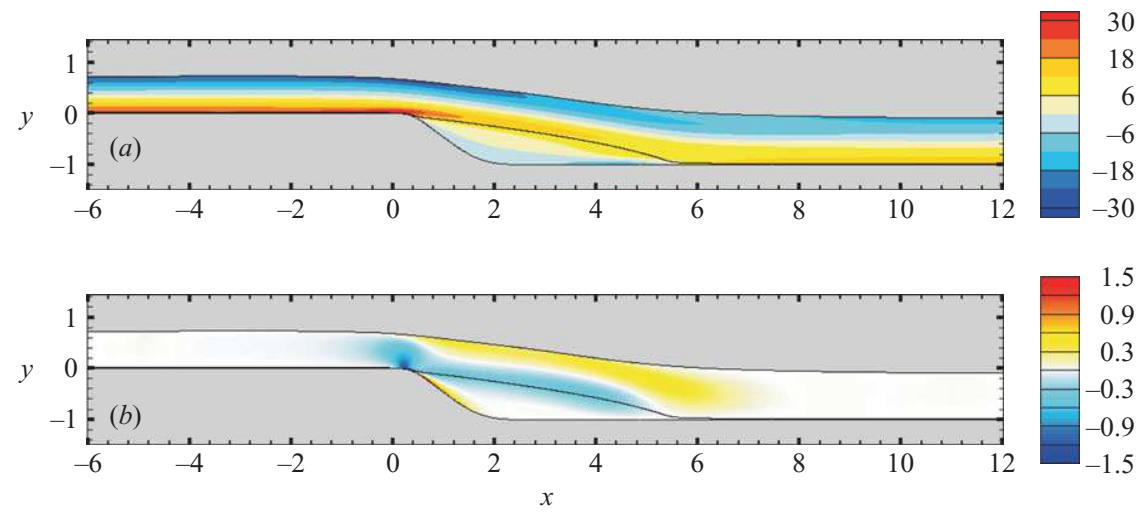

FIgURE 2. Spatial distribution of the derivatives of the base flow. The black line in the flow shows the dividing streamlines linking the separation point to the reattachment point. $(a) \partial_{y} U$, (b) $\partial_{x} V$. Parameter settings: $R e=800$, mesh $M_{b 1}$.

ordered with the same rule as the direct ones, are the complex conjugates of a direct eigenvalue $\left(\sigma_{l}^{+}=\sigma_{l}^{*}\right)$ and that the direct and adjoint global modes respect the bi-orthogonality condition:

$$
\left(\hat{\boldsymbol{q}}_{m}^{+}, \boldsymbol{B} \cdot \hat{\boldsymbol{q}}_{l}\right)=C_{l} \delta_{l m},
$$

which indicates that a particular adjoint global mode indexed by $m$ is orthogonal to all the direct global modes indexed by $l$, except the one associated with the same eigenvalue. From the expressions for $\boldsymbol{A}$ and $\boldsymbol{A}^{+}$(2.6)-(2.12), we easily verify that $\boldsymbol{A}$ and $\boldsymbol{A}^{+}$do not commute, meaning that direct and adjoint global mode bases are not identical and implying that for at least one $l$

$$
\left(\hat{\boldsymbol{q}}_{l}^{+} \boldsymbol{B} \cdot \hat{\boldsymbol{q}}_{l}\right) \neq 1
$$

if both $\hat{\boldsymbol{q}}_{l}$ and $\hat{\boldsymbol{q}}_{l}^{+}$are normalized to unit energy. Properties (2.15) and (2.16) imply that direct eigenmodes are not normal to each other and therefore the energy of a perturbation is not the sum of the squared coefficients of its decomposition onto the base of eigenmodes. This offers the possibility of a transient growth of the perturbation energy even if all the coefficients of the decomposition into eigenmodes decrease in time as is the case for stable flows. For parallel shear flows, transient growth is associated in particular with the lift-up mechanism, i.e. the creation of a large streamwise velocity perturbation through transport of the base-flow momentum by a cross-stream velocity perturbation (see Schmid \& Henningson 2001). In the present formalism, this mechanism corresponds to the off-diagonal terms of $\boldsymbol{A}$ that reduce to $-\partial_{y} U$ if the $V$ component of the base flow is negligible, as is the case when the flow is parallel. This lift-up term transposes from the $u$ to the $v^{+}$component when comparing $\boldsymbol{A}$ and $\boldsymbol{A}^{+}$. As a result of the lift-up, the direct mode presents large values of $u^{\prime}$ whereas the adjoint mode should have large $v^{\prime}$. As seen on figure 2, the lift-up term $-\partial_{y} U$ dominates $-\partial_{x} V$ by two orders of magnitude everywhere in the flow except in the close vicinity of the separation point where the ratio increases to one order of magnitude. This non-normal effect, similar to the lift-up, is local and non-isotropic since at each location in the flow the direct $\boldsymbol{A}$ and adjoint $\boldsymbol{A}^{+}$operators differ by off-diagonal terms and therefore affect the components of the vector differently. By extension this effect coming from the transport of the base flow by the perturbation will be referred to hereafter as lift-up non-normality even though it also accounts 
for the Orr and the Kelvin-Helmholtz mechanisms, in particular when $k=0$. As suggested by Chomaz (2005) in open flow a second type of non-normality arises from the transport of the perturbation by the base flow and complements the lift-up non-normality coming from the base flow transport. This convective non-normality is here expressed in the convective term $C$ that is diagonal and therefore respects isotropy but changes sign between the direct $\boldsymbol{A}$ and adjoint $\boldsymbol{A}^{+}$operators. Physically, it corresponds to downstream transport for the direct perturbations and upstream transport for the adjoint perturbations. As noticed by Chomaz et al. (1990) when analysing the Ginzburg-Landau model equation, this non-normality is specific to open flows and tends to spatially separate the perturbation velocity fields, downstream for the direct perturbations and upstream for the adjoint perturbations.

\section{Numerical resolution}

The two-dimensional stationary Navier-Stokes equations (2.3), and the direct (2.5) and adjoint (2.14) generalized eigenvalue problems are numerically solved by a finiteelement method. The spatial discretization is a mixed finite-element formulation using P2-P1 Hood-Taylor elements: six-node quadratic triangular elements with quadratic interpolation for velocities (P2) and three-node linear triangular elements for pressure (P1) (see Ding \& Kawahara 1999 for details). The meshes as well as the discrete matrices resulting from the variational formulation of the problems (2.3), (2.5) and (2.14) projected onto those meshes are generated with the software FreeFem++ (http://www.freefem.org).

\subsection{Base flow}

To compute the base flow, we proceed as in Barkley et al. (2002): a time-dependent simulation of the two-dimensional Navier-Stokes equations is first used to obtain an approximate solution of the steady solution at low Reynolds number where it is stable to two-dimensional perturbations. A Newton iteration method is then applied to solve the stationary Navier-Stokes equations (2.3), starting the procedure with the approximate steady solution as a guess. The Newton algorithm requires the inversion of a non-symmetrical sparse matrix of size $N_{b} \times N_{b}$ where $N_{b}=2 N_{2 b}+N_{1 b}$ is the total number of nodes and $N_{2 b}$ and $N_{1 b}$ denote respectively the number of nodes associated with the P2 and P1 elements. This inversion is carried out by a direct method, the UMFPACK library being used to perform a sparse LU factorization. Solution at larger Reynolds number is followed by continuity using the Newton method and starting the iterative process with the solution at smaller Reynolds number as a guess. Steady solutions may be computed for any Reynolds number by this procedure even when the flow is unstable in two dimensions. Figure 1(a) shows streamlines of the base flow computed for $R e=800$ which gives a qualitative representation of the recirculation bubble. The skin-friction coefficient $C_{f}$ is defined here as

$$
C_{f}(x)=-\frac{2}{R e} \boldsymbol{t} \cdot\left(\nabla \boldsymbol{U}+\nabla \boldsymbol{U}^{t}\right) \cdot \boldsymbol{n}
$$

where $\boldsymbol{t}$ and $\boldsymbol{n}$ are respectively the tangent oriented downstream and the normal oriented outward from the solid walls. The skin-friction coefficients on the lower and upper walls are respectively depicted in figure 1 by the solid and dashed lines. On the lower wall the skin friction changes sign inside the recirculation bubble. The separation and reattachment points are located at the abscissae $x_{s}=0.46$ and $x_{r}=5.50$, for which the skin friction coefficient is nil (vertical lines). Since the reattachment length 


\begin{tabular}{lcccccc}
\hline Mesh & $L_{o}$ & $N_{2 b}$ & $N_{1 b}$ & $x_{s}$ & $x_{r}$ & $L_{b}$ \\
$M_{b 3}$ & 14 & 102201 & 25874 & 0.46152 & 5.50580 & 5.04428 \\
$M_{b 2}$ & 14 & 185722 & 46884 & 0.46108 & 5.50567 & 5.04459 \\
$M_{b 1}$ & 14 & 256572 & 64699 & 0.46107 & 5.50553 & 5.04446 \\
$L_{b 1}$ & 22 & 258342 & 65164 & 0.46052 & 5.50571 & 5.04519
\end{tabular}

TABLE 1. Results of the convergence test on the base-flow calculations. $R e=800$.

\begin{tabular}{lccccc}
\hline Mesh & $N_{2 s}$ & $N_{1 s}$ & $\sigma_{1}$ & $\sigma_{2 r}$ & $\sigma_{2 i}$ \\
$M_{s 3}$ & 94613 & 23978 & 0.00344581 & -0.04097234 & -0.10552459 \\
$M_{s 2}$ & 125580 & 31775 & 0.00344550 & -0.04097274 & -0.10552477 \\
$M_{s 1}$ & 145602 & 36836 & 0.00344539 & -0.04097288 & -0.10552485 \\
$L_{s 1}$ & 142736 & 36135 & 0.00345051 & -0.04096535 & -0.10553184
\end{tabular}

TABLE 2. Results of the convergence test on the stability calculations. $R e=800, k=1.92$.

is known to be particularly sensitive to the numerical resolution, it has been used to check the convergence of the base-flow calculations when the grid resolution and the dimensions of the computational domain $\Omega$ are varied. The convergence with respect to grid resolution has been checked on the meshes denoted $M_{b 1}$ to $M_{b 3}$ in table 1 . The reference mesh being the low-resolution grid $M_{b 3}$, the convergence test consisted of doubling the number of points close to the separation $\left(M_{b 2}\right)$ and reattachment points $\left(M_{b 1}\right)$. The convergence with respect to external dimensions has been checked by varying the distance $L_{o}$ of the outlet boundary. In table 1 it corresponds to the meshes denoted by $M_{b 1}$ and $L_{b 1}$. The results of the convergence test are reported in table 1 and show that the low-resolution grid $M_{b 3}$ is sufficiently accurate to compute the separation bubble properly.

\subsection{Stability calculations}

Once the base flow $\boldsymbol{Q}$ has been computed on the mesh $M_{b}$, it is then interpolated on a coarser mesh $M_{s}$ on which the three-dimensional stability problems (2.5) and (2.14) are solved. The size of the discrete matrix is now $N_{s} \times N_{s}$ where the total number of nodes is $N_{s}=3 N_{2 s}+N_{1 s}$ and $N_{2 s}$ and $N_{1 s}$ denote respectively the number of nodes associated with the $\mathrm{P} 2$ and $\mathrm{P} 1$ elements of the mesh $M_{s}$. To compute the eigenvalues of largest real part, which are responsible for the bifurcation, a shift-andinvert strategy is used as in Ehrenstein \& Gallaire (2005). The resulting generalized eigenvalue problems are then solved using the Implicitly Restarted Arnoldi method of the library. Since the bifurcation is found to be steady as in Barkley et al. (2002), a real shift parameter has been used in most of the computations. However complex shift parameters have also been investigated to obtain the spectrum along the imaginary axis in the $\left(\sigma_{i}, \sigma_{r}\right)$-plane. By doing this, we checked that all eigenvalues associated with non-zero frequency are stable. For each value of the transverse wavenumber $k$, the generalized eigenvalue problem is solved by computing a selection of about 20 leading eigenvalues. A typical example of the computed eigenvalue spectrum is depicted in figure $3(a)$. All the values plotted are independent of the mesh used and are therefore not spurious. As for the base-flow computation, convergence tests with varying grid resolution $M_{s}$ and domain size $L_{s}$ have been performed. The corresponding two leading eigenvalues $\sigma_{1}$ (real) and $\sigma_{2}$ (complex) are reported in table 2 for the different meshes. Only the fifth digit varies on the grid, showing that the grid $M_{s 3}$ is accurate enough for the stability computation. The leading eigenvalue $\sigma_{1}$, denoted by an open 

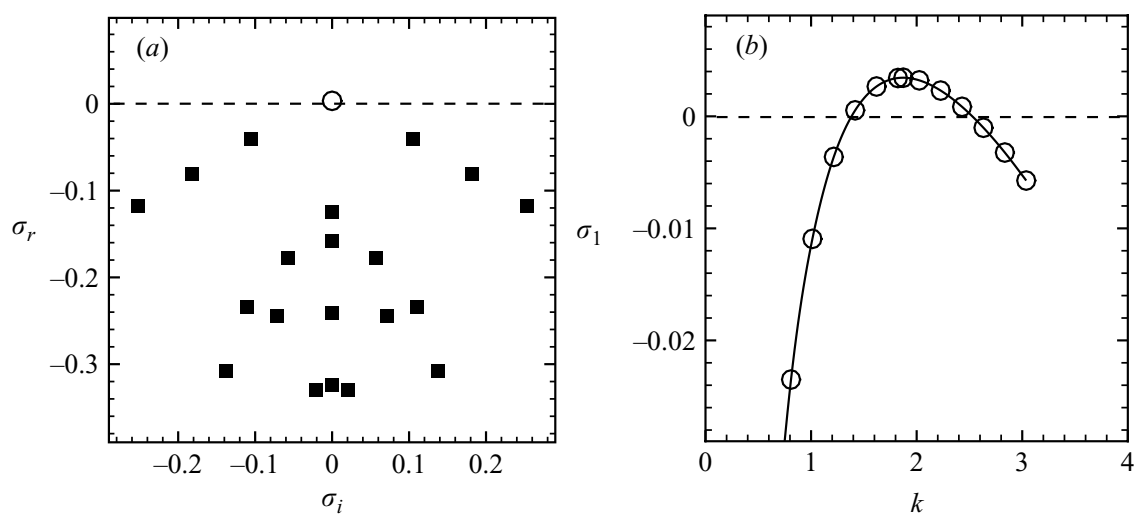

FIGURE 3. Results of the stability calculations. (a) Typical eigenvalue spectrum in the $\left(\sigma_{i}, \sigma_{r}\right)$-plane for the transverse wavenumber $k=1.92$, the base flow being first computed on the mesh $M_{b 1}$ and then extrapolated on the mesh $M_{s 1}$. Open circle indicates the eigenvalue of maximal temporal growth rate $\sigma_{1}$ at this wavenumber $k$. (b) Temporal growth rate of the leading eigenmode $\sigma_{1}$ (in the present case $\sigma_{1}$ is real) as a function of the transverse wavenumber $k$. Parameter settings: $R e=800$.

circle in figure $3(a)$, is plotted as a function of the transverse wavenumber $k$ on figure 3(b) for the Reynolds number value $R e=800$. For high $(k>2.5)$ and low $(k<1.5)$ values of the transverse wavenumber, all the eigenmodes are damped. On the contrary, for intermediate values of $k, \sigma_{1}$ is positive indicating that this wavenumber is unstable. The maximum temporal growth rate $\sigma_{1 \max }=0.00345$ is reached for the transverse wavenumber value $k_{1}=1.92$ which corresponds to transverse wavelength $L_{z}=2 \pi / k_{1}=3.27$. The associated mode is therefore three-dimensional; it is also non-oscillating since $\sigma_{1 i}=0$ and will be referred to as the leading global mode. Hereafter we will focus our attention on this leading mode, considering that all initial perturbations must converge towards it at large time. Its velocity components, described in the next section, have a spatial structure very similar to the leading global mode found in other separated flows as discussed by Barkley et al. (2002) in the case of a backward-facing-step flow and by Gallaire et al. (2005) in the case of a detached boundary layer over a bump.

To determine the leading adjoint eigenmode, we solve the adjoint problem (2.14) for the value of the transverse wavenumber $k_{1}=1.92$ by computing a selection of leading eigenvalues using the same procedure as for the direct problem resolution. Therefore the numerical implementation of the differential operators $\boldsymbol{A}$ and $\boldsymbol{A}^{+}$leads to discrete operators that are not adjoint to each other. Note that in the computation we do not assume that the adjoint eigenvalues $\sigma_{l}^{+}$are complex conjugated with the direct eigenvalues $\sigma_{l}$. This property and the bi-orthogonality relation (2.15) are $a$ posteriori used to check that our numerical procedure accurately estimates the direct and adjoint global modes. We find that the adjoint eigenvalue with the largest real part is $\sigma_{1}^{+}=0.00344540$ (computed on the mesh $M_{s 3}$ ), the difference with $\sigma_{1}$ being of order $10^{-7}$, and that $\left(\hat{\boldsymbol{q}}_{l}, \boldsymbol{B} \cdot \hat{\boldsymbol{q}}_{m}^{+}\right)$is smaller than $10^{-6}$ for the 20 leading normed modes.

\section{Global and adjoint modes}

Figure 4(a) represents the amplitude of the direct global mode defined as the square-root of the energy density field. The separation line of the base flow is also plotted (black curve) in order to compare the position of the global mode 

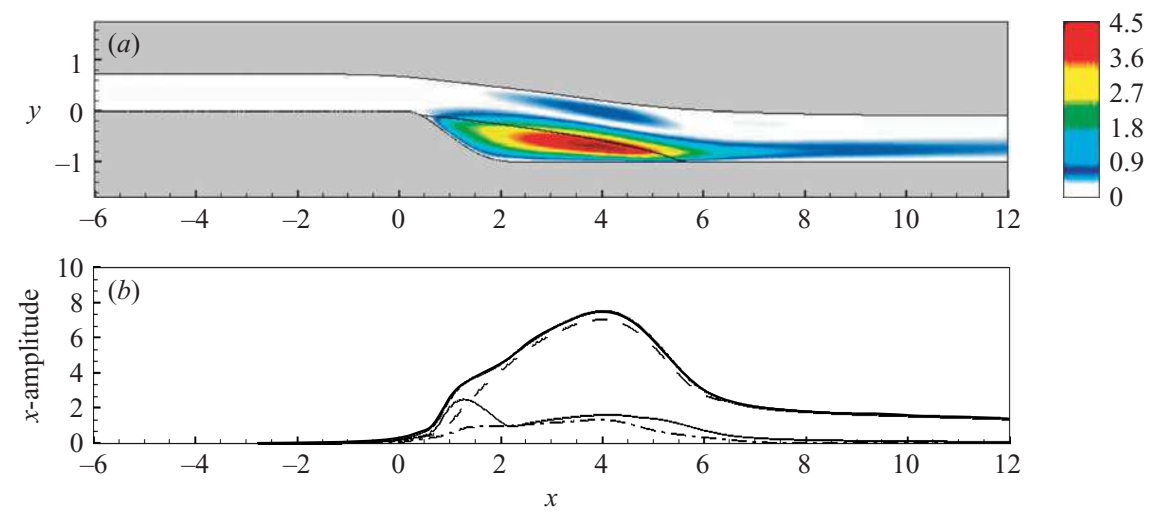

FIGURE 4. Leading direct global mode. (a) Spatial distribution of the velocity magnitude $\left|\hat{\boldsymbol{u}}_{\mathbf{1}}\right|$. The separation line is also shown by a black line. (b) Magnitude of the velocity and of its components integrated over $y:-\left(\int\left|\hat{\boldsymbol{u}}_{1}\right|^{2} \mathrm{~d} y\right)^{1 / 2},---\left(\int\left|\hat{u}_{1}\right|^{2} \mathrm{~d} y\right)^{1 / 2},-\left(\int\left|\hat{v}_{1}\right|^{2} \mathrm{~d} y\right)^{1 / 2}$, $-\cdot-\left(\int\left|\hat{w}_{1}\right|^{2} \mathrm{~d} y\right)^{1 / 2}$, as a function of the longitudinal position $x$. Parameter settings: $R e=800$, $k=1.92$

with the recirculation bubble. The amplitude of the direct global mode reaches a maximum slightly below the free shear layer detached from the separation point and slightly upstream of the reattachment point. It also extends downstream from the recirculation bubble. The amplitude of the direct global mode versus $x$ - which will be called hereafter the $x$-amplitude - is computed as the square-root of the density energy integrated over a vertical cross-section for each streamwise position and is presented on figure $4(b)$ as a thick solid line. The thin dashed, dashed-dotted and solid lines in figure $4(b)$ represent respectively the contribution of the longitudinal, vertical and transverse velocity components to this $x$-amplitude distribution of the direct global mode. Three zones can be distinguished on this figure. In the first zone extending from the inlet to nearly the separation point $\left(x<x_{s}\right)$ the direct global mode is almost nil. In the second region extending from the separation point $\left(x \geqslant x_{s}\right)$ to nearly the reattachment point $\left(x=x_{r}\right)$ the global mode amplitude is maximum. The maximum $x$-amplitude is reached close to the longitudinal location $x=4$. In the third region that extends down from the reattachment point to the outlet, the $x$-amplitude is one order of magnitude smaller than its maximum value and decreases extremely slowly in $x$. Strikingly the $u$-contribution to the direct global mode dominates the entire field except in the close vicinity of the separation point. This suggests that the lift-up mechanism is predominant in the energy production of the direct global mode, as exemplified below.

Figure 5(a) presents a three-dimensional view of the fictitious flow $\boldsymbol{Q}+\beta \boldsymbol{q}_{\mathbf{1}}{ }^{\prime}$ defined as the base flow perturbed by the global mode with an arbitrary finite amplitude $\beta$. This procedure was classically used in mixing layer or jet stability analysis to characterize the physical mechanism underlying the instability (see Maslowe \& Kelly 1970). The flow is viewed from downstream, the spanwise direction is from right to left and its extent corresponds to two wavelengths of the global mode. Isovalues of the streamwise component of the skin friction $C_{f}$ of the total flow are plotted for the lower wall. Note that in this three-dimensional flow the skin-friction coefficient is no longer a scalar but a vector that may be decomposed into a spanwise and a streamwise component. However, since the streamwise component is dominant over the transverse component by one order of magnitude almost everywhere on the walls, we will hereafter identify the 
(a)

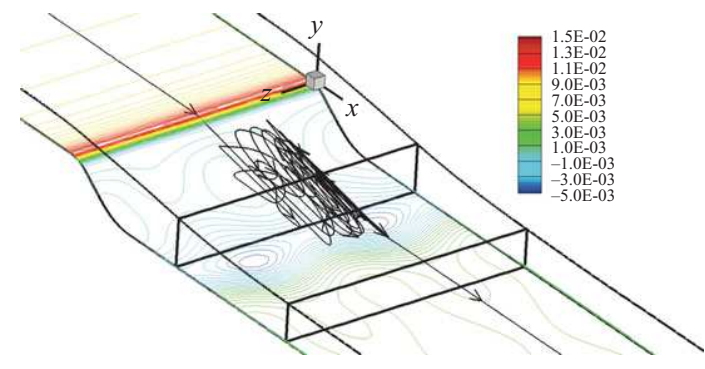

(b)

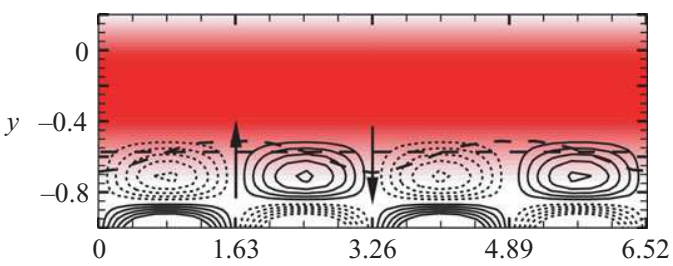

(d)

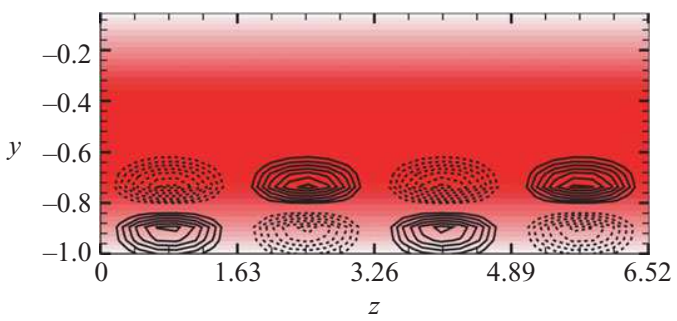

(c)

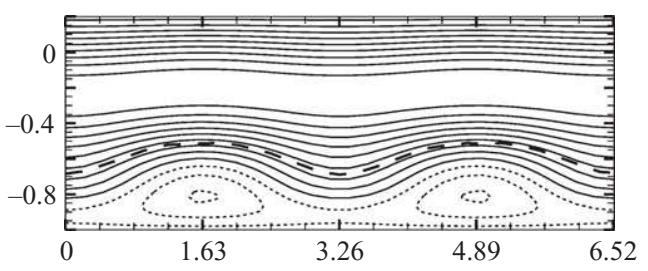

$(e)$

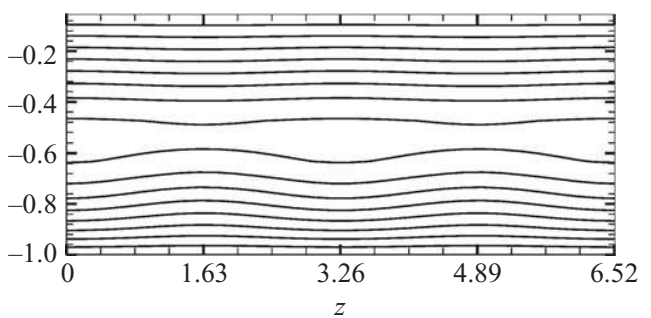

Figure 5. Three-dimensional view of the total flow $Q+\beta \boldsymbol{q}_{1}{ }^{\prime}$ where $\beta$ is an arbitrary finite amplitude here chosen equal to $\beta=0.05$. The visualized portion of the flow extends two global mode wavelengths along the span. The streamwise component of the total skin-friction on the lower wall is indicated by coloured isolines with negative values in blue. A particular three-dimensional streamline is depicted by the solid black line. Cross-stream sections upstream of the reattachment, at $x=4$, and downstream, at $x=8$, are indicated as long rectangles in $(a)$. Fields are shown in $(b)$ and $(c)$ for $x=4$ and in $(d)$ and $(e)$ for $x=8 .(b, d)$ Contours of the streamwise vorticity (dotted lines standing for negative values) with superimposed streamwise velocity of the base flow visualized by colours, from white (negative values) to red (high positive values). In $(b)$ a particular contour $(U=0.298)$ corresponding to the separation line of the base flow is indicated by a horizontal dashed line. $(c, e)$ Contours of the streamwise velocity of the total flow (dotted lines stand for negative values). The dashed line marks the edge of the deformed separation bubble (it is also plotted in $(b)$ for comparison). Parameter settings: $R e=800, k_{1}=1.92, L_{z}=3.26, \beta=0.05$.

skin friction with its streamwise component. A particular three-dimensional streamline of the total flow is also depicted in figure 5(a) by a solid black line. The locations of two cross-stream sections respectively upstream and downstream of the reattachment line are shown by elongated rectangles. Figure $5(b, d)$ depicts the total streamwise vorticity in these two planes and figure $5(c, e)$ depicts the total streamwise velocity. Starting the description of the figure 5(a) from upstream, the skin friction on the lower wall has no transverse variation. The three-dimensional global mode hardly alters the base flow in this region and has approximately no effect on the position of the separation line 

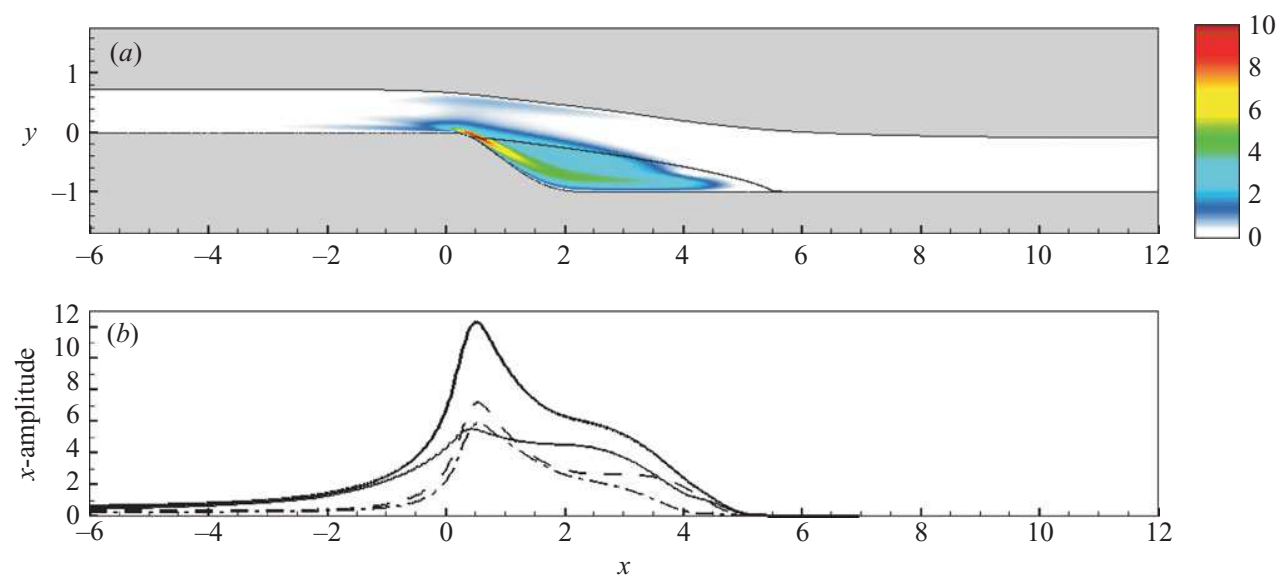

Figure 6. As figure 4 but for the leading adjoint global modes $\hat{\boldsymbol{q}}_{1}^{+}$.

marked by the inversion of the sign of the skin friction along $x$. Inside the recirculation bubble is a dead-flow region characterized by low negative values of the skin friction. The skin friction of the total flow is altered in the reattachment region and the reattachment line is displaced alternately downstream and upstream. Downstream of the reattachment line the deformation of skin friction remains. Higher values correspond to high-speed streaks (see the cross-section in figure 5e). In figure 5(a) the streamline indicates the trajectory of a particular particle released close to the symmetry plane of the perturbation, one period $L_{z}$ away from $z=0$. Once it enters the recirculation bubble the particle circles around several times before exiting the bubble with a lateral displacement of half a period, at a position near where the reattachment line is most displaced downstream. In figure $5(b)$ are depicted the streamwise vorticity of the total flow (isocontours) and the streamwise velocity of the base flow (shading) in the cross-stream section located upstream from the reattachment line (see figure $5 a$ ). The base flow being two-dimensional, the streamwise vorticity is that of the perturbation flow. Weak self-sustained streamwise vortices depicted by the isocontours are generated inside the shear layer of the recirculation bubble. These streamwise vortices are of opposite sign and thus induce alternate vertical velocity perturbations. For instance the vertical velocity perturbation, represented schematically by arrows on figure $5(b)$, is positive in the half-wavelength plane $(z=1.63)$ and negative in the wavelength plane $(z=3.26)$. In the half-wavelength plane low-speed fluid in the shear layer of the recirculation bubble (white region) is transported by the vertical velocity perturbation towards regions of higher speed in the shear layer (red regions in figure $5 b$ ), and vice versa in the wavelength plane. This lift-up mechanism thus generates low- and high-speed streaks in the longitudinal direction. A typical pattern of those streaks in a cross-section is shown in figure $5(c)$ where the $u$-velocity of the total flow is plotted. The dotted and solid lines show respectively negative and positive isovalues of the streamwise velocity. This deformation of the recirculation bubble has the effect of displacing upstream and downstream the reattachment line (see figure $5 a$ ). Downstream of the reattachment line a similar but weaker lift-up mechanism is active with weak streamwise vortices (see figure $5 c$ ) associated with low- and high-speed streaks (see figure $5 e$ ). On both figures $5(b)$ and $5(d)$ streamwise vortices are separated from the wall by opposite sign vorticity at the wall.

We turn now to the characteristics of the adjoint global mode $\hat{\boldsymbol{q}}_{1}^{+}$depicted in figures $6(a)$ and $6(b)$ which show respectively the amplitude of the adjoint global 
mode $\left|\hat{\boldsymbol{u}}_{1}^{+}\right|$in the computational domain and its $x$-amplitude (i.e. $\left.\left(\int\left|\hat{\boldsymbol{u}}_{1}^{+}\right|^{2} \mathrm{~d} y\right)^{1 / 2}\right)$ as a function of the streamwise position $x$. Like the direct global mode, three regions can be identified. Since the advection by the base flow is reversed in the adjoint evolution operator $\boldsymbol{A}^{+}$we start the description from downstream. From the outlet to the reattachment point the adjoint global mode is nil. It is most intense in the recirculation bubble and reaches its maximum at the separation point. Upstream of that point its $x$-amplitude is one order of magnitude smaller and concentrated on the $w+$ component. A comparison of figures 4 and 6 indicates a spatial separation of the direct and adjoint global modes related to the convective non-normality due to the base-flow advection as discussed in $\S 2.3$. The direct global mode is nil upstream of the separation and the adjoint is nil downstream of the reattachment. Both the direct and adjoint global modes are maximum inside the recirculation bubble and only their maxima are separated. The direct mode is maximum close to the reattachment and the adjoint is maximum close to the separation.

The direct and adjoint global modes also reflect characteristics of the lift-up nonnormality since, as discussed in $\S 2$, the direct mode is dominated by streamwise perturbation (figure 4) and the adjoint mode by cross-stream perturbation (figure 6), respectively downstream of the reattachment point and upstream of the separation point. Close to the reattachment and separation points, all the components contribute to the dynamics of both the direct and adjoint global modes, suggesting a close interplay between convective and lift-up non-normalities.

As discussed in Chomaz (2005), close to the bifurcation the leading global mode defines the slow manifold and on that slow time scale the adjoint global mode characterizes the sensitivity to initial conditions, the receptivity to forcing and the sensitivity to base flow modification.

An initial perturbation $\hat{\boldsymbol{q}}_{0}$ may be decomposed on the eigenmode base and the coefficient of this decomposition on a particular mode is equal to the scalar product of this initial perturbation with the adjoint of that mode, divided by the scalar product of that mode with its adjoint:

$$
A_{l}=\frac{\left(\boldsymbol{B} \cdot \hat{\boldsymbol{q}}_{l}^{+}, \hat{\boldsymbol{q}}_{0}\right)}{\left(\hat{\boldsymbol{q}}_{l}^{+}, \boldsymbol{B} \cdot \hat{\boldsymbol{q}}_{l}\right)} .
$$

It depends only on the direct and adjoint velocities and not on the associated pressure field. Before or at the bifurcation the flow converges at large time towards the leading global mode, so that only the coefficient $A_{1}$ of this decomposition for the leading global mode $\hat{\boldsymbol{q}}_{1}$ is of interest (see Schmid \& Henningson 2001). If the direct and adjoint global modes are of unit norm, the coefficient $A_{1} \exp \left[\sigma_{1} t\right]$ represents the amplitude of the global mode at large time. For an initial perturbation localized in space (for example of delta function form $\delta\left(x-x_{0}, y-y_{0}\right)$ ), the coefficient $A_{1}$ is the value of the adjoint global mode at $\left(x_{0}, y_{0}\right)$. The spatial distribution of the adjoint global mode shows the region of the flow where the global mode is sensitive (or receptive) to a localized initial perturbation. From figure $6(b)$ we see that the sensitivity to the initial perturbation is the highest close to the wall inside the recirculation bubble and reaches a maximum in the vicinity of the separation point. From (4.1), the most dangerous initial perturbation, i.e. the initial perturbation of unit norm that leads to the highest global mode amplitude, is the adjoint global mode $\left(\hat{\boldsymbol{q}}_{0}=\hat{\boldsymbol{q}}_{1}^{+}\right)$. Figure $7(a)$ is a magnified visualization of the adjoint global mode in the region of the separation point where the dividing streamline of the recirculation bubble is depicted by the thick dashed line. The streamlines show that the direction of the optimal initial 
(a)

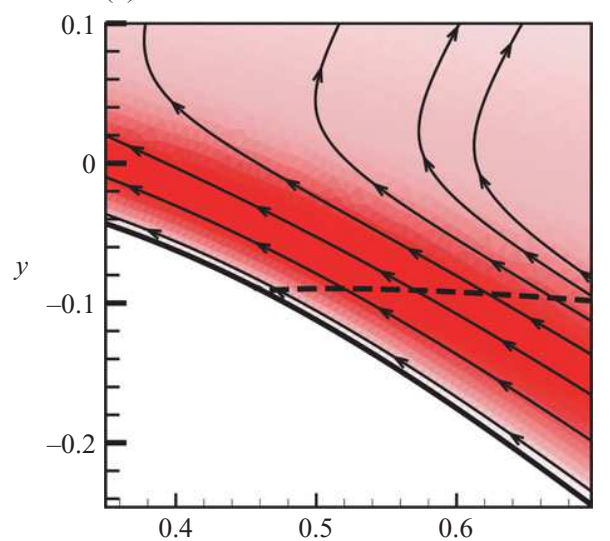

(c)

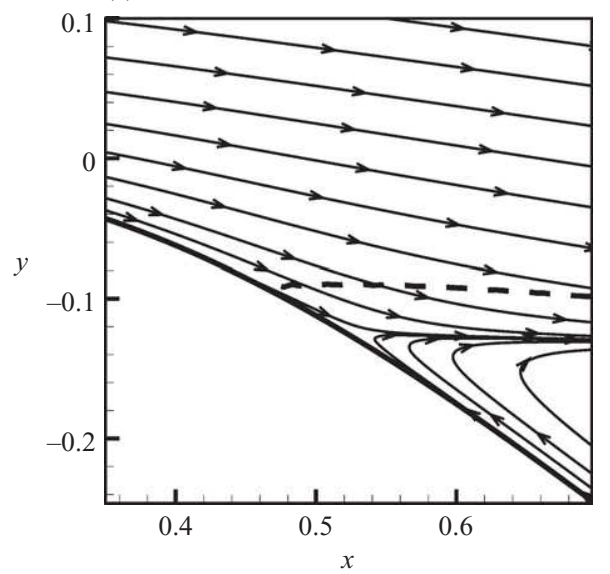

(b)

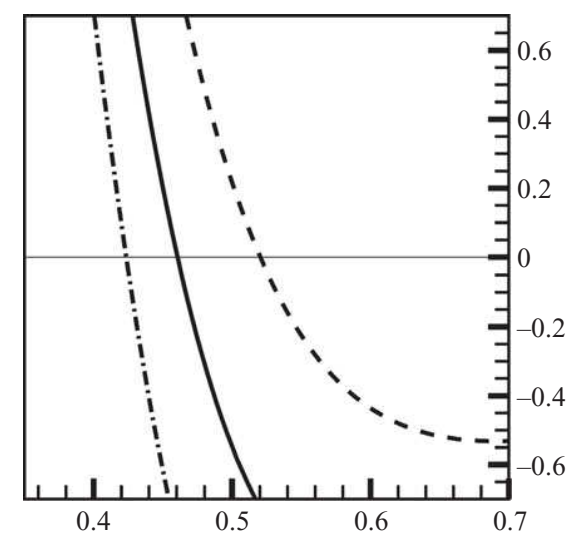

(d)

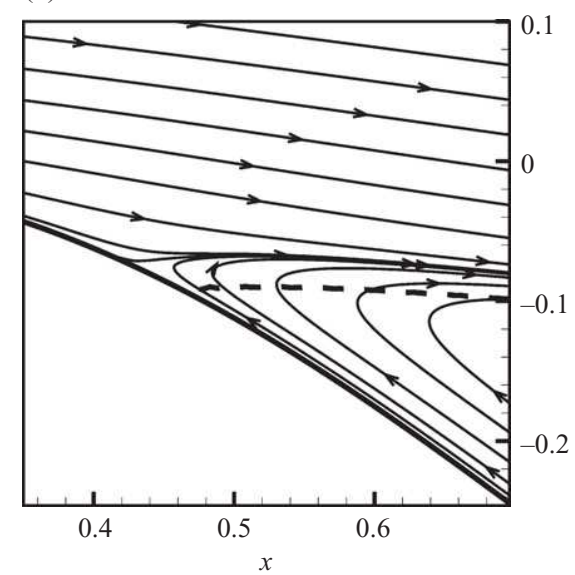

FIGURE 7. Physical interpretation of the optimal initial perturbation. (a) Magnified visualization of the adjoint velocity field $\hat{\boldsymbol{u}}_{1}^{+}$close to the separation point in a spanwise plane one period $L_{z}$ away from $z=0$. Red and white regions correspond respectively to high and low values of the amplitude of the adjoint global mode, its orientation being shown by the streamlines. $(c, d)$ Streamlines of the total flow $\boldsymbol{q}=\boldsymbol{Q}+\alpha\left(\hat{\boldsymbol{q}}_{1}^{+} \exp \left[\mathrm{i} k_{1} z\right]+\right.$ c.c. $)$ close to the separation point in spanwise planes $(c)$ half a period $L_{z} / 2$ and $(d)$ one period $L_{z}$ away from $z=0$. (b) Streamwise component of the skin-friction coefficient of the total flow as a function of the longitudinal position $x$ for three spanwise planes. Dashed line, $z=L_{z} / 2$; solid lines, $z=3 L_{z} / 4$; dashed-dotted line $z=L_{z}$. The dashed lines in $(a),(c)$ and $(d)$ show the separation line of the unperturbed flow, i.e. the base flow. Parameter settings: $R e=800$, $k=k_{1}, \alpha=0.001$.

perturbation, close to the separation point, is parallel to the lower wall and oriented downstream. The strength of this vector field is indicated by the colour shading where red regions denote high perturbation velocities. The effect of the optimal initial perturbation may be physically interpreted by considering, as we did for the direct global mode, the flow reconstructed as the linear superposition of the base flow $Q$ and the adjoint global mode $\hat{\boldsymbol{q}}_{1}^{+}$with an arbitrary finite amplitude $\alpha$. Figure $7(c, d)$ depicts the streamlines of the total flow close to the separation point in two spanwise planes with opposite phase $z=L_{z} / 2$ and $z=L_{z}$. The lower wall is marked by the thick solid line and the dividing streamline of the base flow is the thick dashed line. We see that the separation point is displaced upstream and downstream and the 
recirculation region deflates and inflates as we move along the $z$-axis. This is confirmed by computing the skin-friction coefficient of the total flow, plotted in figure $7(b)$ as a function of the longitudinal coordinate $x$. The solid line corresponds to the skinfriction coefficient on the lower wall in the spanwise plane $z=3 L_{z} / 4$ which, since the longitudinal and vertical velocity components of the optimal perturbation are nil there, is identical to the skin-friction coefficient of the base flow. The dashed and dashed-dotted lines represent respectively the skin-friction coefficient in the planes $z=L_{z} / 2$ and $z=L_{z}$. The optimal initial perturbation, given by the adjoint global mode, physically corresponds to a streamwise displacement of the separation point varying along the span.

For stable flow, the adjoint global mode also quantifies the response to forcing. In the present case, when stationary forcing is considered, the forcing term may be either a momentum or a mass forcing term, the receptivity of the global mode to volumic forcing being evaluated by

$$
A_{f}=\frac{\left(\hat{\boldsymbol{u}}_{1}^{+}, \hat{\boldsymbol{f}}\right)}{\left(\hat{\boldsymbol{q}}_{1}^{+}, \boldsymbol{B} \cdot \hat{\boldsymbol{q}}_{\mathbf{1}}\right)}, A_{m}=\frac{\left(\hat{p}_{1}^{+}, \hat{m}\right)}{\left(\hat{\boldsymbol{q}}_{1}^{+}, \boldsymbol{B} \cdot \hat{\boldsymbol{q}}_{\mathbf{1}}\right)},
$$

where the same notation (, ) is used for the three-dimensional and one-dimensional inner products. $A_{f}$ and $A_{m}$ stand for the amplitude of the global mode $\hat{q}_{1}$ when it is forced by, respectively, a body force $\hat{\boldsymbol{f}}=\left(\hat{\boldsymbol{f}}_{x}, \hat{\boldsymbol{f}}_{y}, \hat{\boldsymbol{f}}_{z}\right)$ or a mass source $\hat{m}$ with the same spanwise wavelength as the global mode. Therefore the velocity field of the adjoint global mode $\hat{\boldsymbol{u}}_{1}^{+}$determines regions of the flow receptive to momentum forcing and the amplitude of the global mode $A_{f}$ will be large, and thus the forcing efficient, when the body force is applied in regions where $\hat{\boldsymbol{u}}_{1}^{+}$is large, here close to the separation point. Equivalently the adjoint pressure field indicates regions of receptivity to mass forcing. Figure 8 depicting the pressure distribution of the leading adjoint global mode indicates that a volumic mass forcing should be placed just upstream of the separation point to have a large impact on the leading global mode.

In the case of forcing through blowing or suction at the wall (or eventually at the inlet), Giannetti \& Luchini (2007) recently expressed the dependence of the global mode amplitude on the velocity $\hat{\boldsymbol{u}}_{w}$ at the wall $\Gamma$, as

$$
A_{w}=\frac{\left\langle\hat{p}_{1}^{+} \boldsymbol{n}+R e^{-1} \nabla \hat{\boldsymbol{u}}_{1}^{+} \cdot \boldsymbol{n}, \hat{\boldsymbol{u}}_{w}\right\rangle_{\Gamma}}{\left(\hat{\boldsymbol{q}}_{1}^{+}, \boldsymbol{B} \cdot \hat{\boldsymbol{q}}_{\mathbf{1}}\right)},
$$

where $\langle., .\rangle_{\Gamma}$ corresponds to integration along the wall

$$
\left\langle\hat{\alpha}, \hat{\alpha}^{\prime}\right\rangle_{\Gamma}=\int_{\Gamma} \hat{\alpha}^{t} \cdot \hat{\alpha}^{\prime} \mathrm{d} \Gamma,
$$

for three-component vector fields $\hat{\alpha}$ and $\hat{\alpha}^{\prime}$.

The amplitude of the global mode $A_{w}$ when forced by a velocity at the wall is determined by the wall pressure $\hat{p}_{1}^{+}$and the velocity gradient tensor $\nabla \hat{\boldsymbol{u}}_{1}^{+}$weighted by the inverse of the Reynolds number. The latter term may be neglected compared to the former when the Reynolds number is large and at $R e=800$ it is already one order of magnitude smaller than the pressure. The orientation of the forcing velocity with respect to the wall also influences the response of the global mode since, when the viscous term in (4.3) is neglected, for a given magnitude of the wall velocity $\left|\hat{\boldsymbol{u}}_{w}\right|$, 

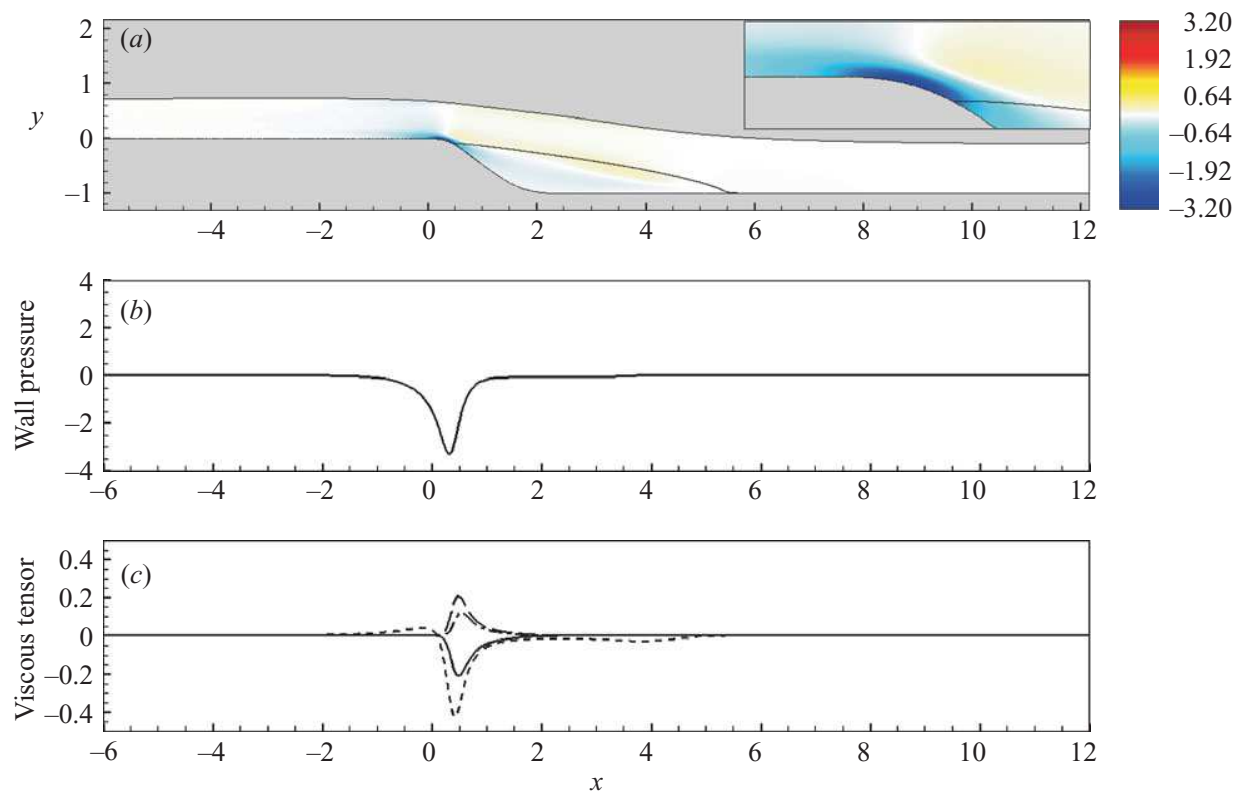

FiguRE 8. Receptivity to mass and boundary forcing. (a) Spatial distribution of the pressure of the adjoint global mode, with a magnified view of the separation region in the top right corner. (b) Wall pressure of the adjoint global mode $\hat{p}_{1}^{+}$on the lower wall as a function of the longitudinal position $x$. (c) Components of the adjoint viscous tensor $R e^{-1} \nabla \hat{\boldsymbol{u}}_{1}^{+}$on the lower wall as a function of the longitudinal position $x .-, R e^{-1} \partial_{x} \hat{u}_{1}^{+},---R e^{-1} \partial_{y} \hat{u}_{1}^{+}$, $-\cdot-R e^{-1} \partial_{x} \hat{v}_{1}^{+},--\longrightarrow, R e^{-1} \partial_{y} \hat{v}_{1}^{+}$. Parameter settings: $R e=800, k=k_{1}$.

the closer the orientation to the normal vector to the wall, the larger the amplitude $A_{w}$. The adjoint pressure and the four components of the velocity gradient tensor weighted by the Reynolds number (the adjoint viscous tensor) are evaluated on the lower wall and respectively plotted in figures $8(b)$ and $8(c)$ as a function of the streamwise position. Upstream from $x=-1$ and downstream from $x=1$ the adjoint pressure $\hat{p}^{+}$is almost zero whereas in the close vicinity of the separation point, it reaches a maximum. The four components of the tensor have a similar shape but their maximum value is one order of magnitude smaller than for the adjoint pressure. At this Reynolds number, the dominant term in evaluating the amplitude of the response of the global mode to a wall velocity forcing is the adjoint wall pressure.

To determine the region of the flow where feedback processes at the origin of a selfsustained instability are active, Chomaz (2005) and Giannetti \& Luchini (2003, 2007) have examined the sensitivity of the leading eigenvalue to structural perturbations of the linear evolution operator (respectively on the Ginzburg-Landau model equation and for the two-dimensional circular cylinder flow configuration). For clarity of presentation, mathematical details of the structural analysis leading to the definition of the sensitivity are given in Appendix B.

The sensitivity $S_{l}$ of a particular eigenvalue $\sigma_{l}$ is defined as a bound of the eigenvalue variation $\left|\delta \sigma_{l}\right|$ which is induced by a local structural perturbation $\delta \boldsymbol{A}_{0}$ of the linearized Navier-Stokes operator (2.6). If structural perturbations of unit norm are considered, the sensitivity is given by (see Appendix B for details)

$$
S_{l}\left(x_{0}, y_{0}\right)=\left|\left(\hat{\boldsymbol{q}}_{l}^{+}, \boldsymbol{B} \cdot \hat{\boldsymbol{q}}_{l}\right)\right|^{-1}\left|\hat{\boldsymbol{u}}_{l}\left(x_{0}, y_{0}\right)\right| \cdot\left|\hat{\boldsymbol{u}}_{l}^{+}\left(x_{0}, y_{0}\right)\right|
$$



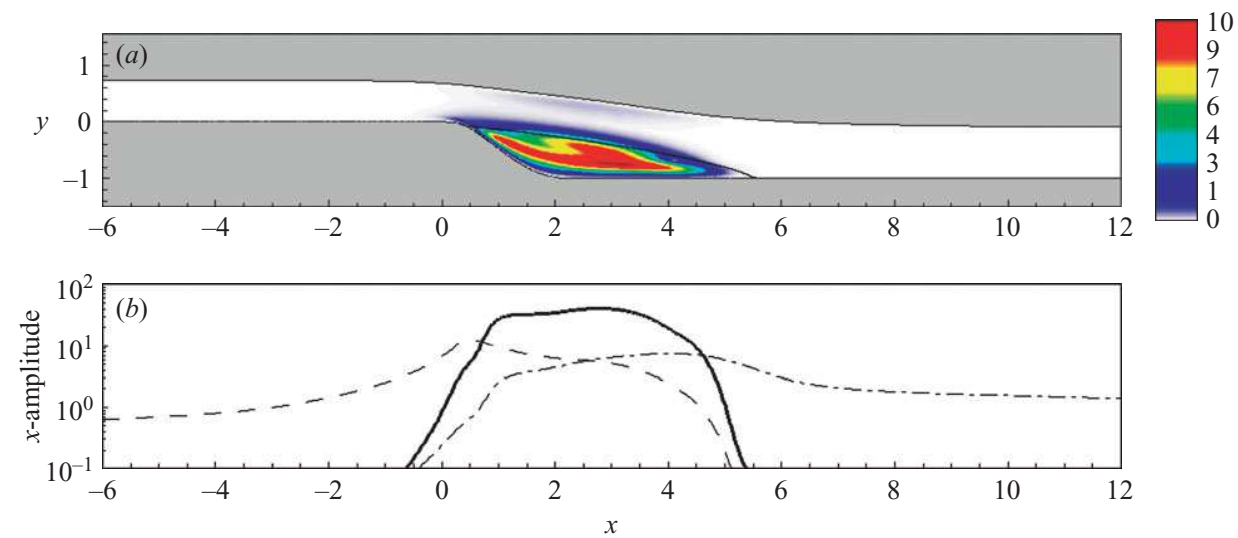

FIGURE 9. Sensitivity to local base flow modifications. (a) Spatial distribution of the sensitivity $\left|\hat{\boldsymbol{u}}_{\mathbf{1}}\right| \cdot\left|\hat{\boldsymbol{u}}_{1}^{+}\right|$, i.e. the amplitude of the direct global mode times the amplitude of the adjoint dominant modes, weighted by the inverse of $\left(\hat{\boldsymbol{q}}_{1}, \boldsymbol{B} \cdot \hat{\boldsymbol{q}}_{1}^{+}\right)$. (b) Sensitivity $\left(\hat{\boldsymbol{q}}_{\mathbf{1}}, \boldsymbol{B} \cdot \hat{\boldsymbol{q}}_{1}^{+}\right)^{-1}\left|\hat{\boldsymbol{u}}_{\mathbf{1}}\right| \cdot\left|\hat{\boldsymbol{u}}_{1}^{+}\right|$ (- ), amplitude of the adjoint global mode $\left|\hat{\boldsymbol{u}}_{1}^{+}\right|(---)$and direct global mode $\left|\hat{\boldsymbol{u}}_{1}\right|$ $(-\cdot)$ integrated in $y$ and plotted as a function of the longitudinal position $x$. Parameter settings: $R e=800, k=k_{1}$

where $\hat{\boldsymbol{q}}_{l}$ and $\hat{\boldsymbol{q}}_{l}^{+}$are the direct and adjoint global modes associated with the eigenvalue $\sigma_{l}$ and $\left(x_{0}, y_{0}\right)$ stands for the spatial location where the structural perturbation is applied. When the linearized Navier-Stokes operator is normal, the sensitivity of any eigenvalue is equal to 1 since the direct and adjoint global modes are identical. The magnitude of the eigenvalue variation induced by the structural perturbation is therefore bounded by the norm of the structural perturbation (here equal to 1). When the linearized Navier-Stokes operator is non-normal, the sensitivity to it may be much larger than 1 and, therefore, the magnitude of the eigenvalue variation may be much larger than the norm of the structural perturbation. The sensitivity defined by (4.5) is the product of two terms. The first term, called the coefficient of non-normality, quantifies the sensitivity of the eigenvalue independently of the location of the structural perturbation. Since the term $\left|\left(\hat{\boldsymbol{q}}_{l}^{+}, \boldsymbol{B} \cdot \hat{\boldsymbol{q}}_{l}\right)\right|$ may be small for non-normal operators, the coefficient of non-normality may be much larger than 1 . The second term quantifies the sensitivity of the eigenvalue as a function of the spatial location $\left(x_{0}, y_{0}\right)$ of the structural perturbation. The sensitivity will be large only if the structural perturbation is applied in the region where the product of the modulus of the direct global mode with the modulus of the adjoint global mode $\left|\hat{\boldsymbol{u}}_{\mathbf{1}}(x, y)\right| \cdot\left|\hat{\boldsymbol{u}}_{1}^{+}(x, y)\right|$ is not zero. For conciseness, this region is now called the overlap region. For open flows, for which the non-normality arises partly from the convective terms (see $\S 2.3$ ), the overlap region is localized in space since the direct and adjoint global modes vanish respectively upstream and downstream.

Giannetti \& Luchini (2007) have determined the sensitivity of the leading eigenvalue in the case of a two-dimensional circular cylinder flow. They have shown that the overlap region is localized in space, just downstream of the cylinder. For the present three-dimensional flow configuration, we have computed the sensitivity of the leading eigenvalue $\sigma_{1}$. The product $\left|\hat{\boldsymbol{u}}_{\mathbf{1}}(x, y)\right| \cdot\left|\hat{\boldsymbol{u}}_{1}^{+}(x, y)\right|$ is depicted in figure $9(a)$. It is almost zero everywhere in the flow, except in the centre of the recirculation bubble where large values are reached. Figure $9(b)$ represents, as a function of the longitudinal position, the $x$-amplitude of the direct (dash-dotted line) and adjoint (dashed line) 
global modes, taken from figures $4(b)$ and $6(b)$, as well as the product of these two quantities (solid line) given by the coefficient of non-normality $\left|\left(\hat{\boldsymbol{q}}_{1}^{+}, \boldsymbol{B} \cdot \hat{\boldsymbol{q}}_{\mathbf{1}}\right)\right|^{-1}$. The $x$-amplitude of the direct (respectively adjoint) global mode vanishing upstream (respectively downstream) of the recirculation bubble, and the overlap region is clearly localized inside the recirculation bubble.

In physical terms, this overlap region is crucial in determining the wavemaker region of a self-sustained hydrodynamic instability, i.e. the region of the flow where the hydrodynamic feedback process is active. Indeed, if we assume that the hydrodynamic feedback process may be modelled by a forcing of the momentum equations $\hat{\boldsymbol{f}}$ proportional to the local instability velocity, i.e. $\hat{\boldsymbol{f}}(x, y)=\delta \boldsymbol{A}_{0} \hat{\boldsymbol{u}}_{\boldsymbol{l}} \delta\left(x-x_{0}, y-y_{0}\right)$, then the sensitivity of an eigenvalue to such a local feedback is given by (4.5) (see Giannetti \& Luchini (2007) for more details). The overlap region depicted in figure 9(a) thus identifies the region of the flow where a local feedback will have a large impact on the leading eigenvalue $\sigma_{1}$. Conversely, any local feedback applied outside this overlap region only slightly modifies this eigenvalue, indicating that these regions do not play a significant role in the process giving rise to the global instability. For the present flow configuration, we may conclude that the recirculation bubble is the wavemaker region of the three-dimensional instability.

\section{Conclusion}

In this paper we have studied the linear dynamics of a two-dimensional flow in an S-shaped duct. A global stability analysis has been performed to account for the non-parallelism of the recirculation bubble and has been numerically solved using a finite-element formulation. The primary bifurcation has been shown to be steady and three-dimensional. A detailed analysis of the structure of the leading global mode suggested that a lift-up mechanism was responsible for the energy production of the instability. The physical effect of this three-dimensional instability on the recirculation bubble has been characterized as a deformation of the reattachment line. The nonnormality of the linearized Navier-Stokes operator has been outlined and discussed in terms of a lift-up non-normality resulting from the transport of the base flow by the perturbation which is characteristic of shear flows, and a convective nonnormality resulting from the transport of the perturbations by the base flow which is characteristic of open flows. By computing the adjoint global mode associated with the direct global mode, this distinction between the two types of non-normality has then been discussed for the present case corresponding to a real open shear flow. The spatial separation of the direct and adjoint global modes has been interpreted as a result of the convective non-normality, whereas the separation in components, the direct global mode being dominated by the streamwise component and the adjoint global mode by crossflow components almost everywhere, as an effect of the lift-up non-normality. A detailed analysis of the adjoint global mode has allowed a complete physical interpretation of the instability close to the bifurcation and the distinguishing of different regions of the flow. The vicinity of the separation point is where the threedimensional global mode is most receptive to initial perturbations and local forcing. This region is distinct from the wavemaker region which has been identified as the whole recirculation bubble by considering the overlap of the direct and adjoint global modes. In the perspective of controlling the instability close to the bifurcation this analysis suggests different locations of the actuator depending on the control method. In the case of a passive control for which a small device is introduced in the flow and induces local modifications of the base flow, it should be placed where both the 
direct and adjoint global modes are not zero, i.e. in the recirculation bubble, to obtain a large impact on the instability development. In the active control framework, the underlying idea being to produce a large effect in the flow by introducing only a small amount of energy, the adjoint global mode is essential (Akervik et al. 2007). For example if the actuator imposes either a small wall displacement or wall blowing and suction, the adjoint pressure field shows that maximum efficiency is achieved by placing the actuator just upstream of the separation point.

\section{Appendix A. Shape of the lower and upper walls}

The shapes of the lower and upper walls are presented in the non-dimensional Cartesian coordinate system. The lower wall is a rounded backward-facing step composed of three different parts:

$$
\begin{aligned}
& y=p 1 l(x)= \frac{A B}{2 h \pi}\left(\sin \left(\frac{h \pi x}{B}\right)-\frac{h \pi x}{B}\right), \quad x \in\left[0, \frac{B}{h}\right], \\
& y=p 2 l(x)=\frac{A B}{2 h}\left(1-\frac{2 h x}{B}\right), \quad x \in\left[\frac{B}{h}, \frac{B}{h}+\frac{M}{h \sqrt{1+A^{2}}}\right], \\
& y=p 3 l(x)=\frac{A C}{2 h \pi}\left(-\sin \left(\frac{\pi}{C}\left(h x-B-\frac{M}{\sqrt{1+A^{2}}}\right)\right)-\frac{\pi}{C}\left(h x-B-\frac{M}{\sqrt{1+A^{2}}}\right)\right) \\
& \quad-\frac{A}{2 h}\left(C+B+\frac{2 M}{\sqrt{1+A^{2}}}\right), \quad x \in\left[\frac{B}{h}+\frac{M}{h \sqrt{1+A^{2}}}, \frac{L}{h}\right] . \quad \text { (A 3) }
\end{aligned}
$$

The step length is denoted by $L$ and the step height by $h$. The value of the parameters used to define the lower wall are $h=0.2025, L=0.5, A=0.75, B=0.15, C=0.31$ and $M=0.31$. In order to design the curved upper wall preliminary calculations have been carried out. A flat plate was first considered as the upper wall and its distance from the lower wall was increased in order to prevent separation from occurring on the flat upper wall. The streamline emerging from the inlet boundary at the vertical coordinate $y=0.723605$ was then extracted and the upper wall finally defined by using interpolated functions of this streamline. The absence of separation on that wall is then verified numerically by computing the skin friction on the upper wall (figure 1) and checking that it never vanishes.

\section{Appendix B. Structural stability and sensitivity}

The structural stability analysis of a linear operator $\boldsymbol{A}$ consists of investigating how structural perturbations of the operator modify its spectrum. Let us consider that $\boldsymbol{A}$ is the linearized Navier-Stokes operator (2.6). Introducing a structural perturbation $\epsilon \delta \boldsymbol{A}$ (of norm $\epsilon$ ) in the perturbation equations (2.5) yields

$$
(\boldsymbol{A}+\epsilon \delta \boldsymbol{A})\left(\hat{\boldsymbol{q}}_{l}+\epsilon \delta \hat{\boldsymbol{q}}_{l}\right)=\left(\sigma_{l}+\epsilon \delta \sigma_{l}\right) \boldsymbol{B}\left(\hat{\boldsymbol{q}}_{l}+\epsilon \delta \hat{\boldsymbol{q}}_{l}\right)
$$

where $\delta \sigma_{l}$ and $\delta \hat{\boldsymbol{q}}_{l}$ are the eigenvalue and eigenvector variations induced by the structural perturbation. If only infinitesimal structural perturbation are considered $\epsilon \ll 1$, we obtain the expression

$$
\left(\boldsymbol{A}-\sigma_{l} \boldsymbol{B}\right) \delta \hat{\boldsymbol{q}}_{l}+\delta \boldsymbol{A} \hat{\boldsymbol{q}}_{l}=\delta \sigma_{l} \boldsymbol{B} \hat{\boldsymbol{q}}_{l}
$$

by using that $\left(\sigma_{l}, \hat{\boldsymbol{q}}_{l}\right)$ solves the perturbations equations $(2.5)$. The above expression is then projected onto the adjoint global mode $\hat{\boldsymbol{q}}_{l}^{+}$

$$
\left(\hat{\boldsymbol{q}}_{l}^{+},\left(\boldsymbol{A}-\sigma_{l} \boldsymbol{B}\right) \delta \hat{\boldsymbol{q}}_{l}\right)+\left(\hat{\boldsymbol{q}}_{l}^{+}, \delta \boldsymbol{A} \hat{\boldsymbol{q}}_{l}\right)=\delta \sigma_{l}\left(\hat{\boldsymbol{q}}_{l}^{+}, \boldsymbol{B} \hat{\boldsymbol{q}}_{l}\right) .
$$


The first term on the left-hand side of (B3) is equal to zero:

$$
\left(\hat{\boldsymbol{q}}_{l}^{+},\left(\boldsymbol{A}-\sigma_{l} \boldsymbol{B}\right) \delta \hat{\boldsymbol{q}}_{l}\right)=(\underbrace{\left(\boldsymbol{A}^{+}-\sigma_{l}^{*} \boldsymbol{B}\right) \hat{\boldsymbol{q}}_{l}^{+}}_{=0}, \delta \hat{\boldsymbol{q}}_{i})
$$

since the adjoint global mode $\hat{\boldsymbol{q}}_{l}^{+}$solves the adjoint equation perturbations (2.14). The eigenvalue variation is then related to the structural perturbation $\delta \boldsymbol{A}$ through

$$
\delta \sigma_{l}=\frac{\left(\hat{\boldsymbol{q}}_{l}^{+}, \delta \boldsymbol{A} \cdot \hat{\boldsymbol{q}}_{l}\right)}{\left(\hat{\boldsymbol{q}}_{l}^{+}, \boldsymbol{B} \cdot \hat{\boldsymbol{q}}_{l}\right)} .
$$

Let us now consider a specific structural perturbation $\delta \boldsymbol{A}$, which is spatially localized in $\left(x_{0}, y_{0}\right)$ and acts only on the momentum equations, i.e.

$$
\delta \boldsymbol{A}(x, y)=\left(\begin{array}{cc}
\delta \boldsymbol{A}_{0} & 0 \\
0 & 0
\end{array}\right) \delta\left(x-x_{0}, y-y_{0}\right)
$$

where $\delta$ is the Kronecker symbol, and $\delta \boldsymbol{A}_{0}$ is the local structural perturbation acting on the momentum equations. The magnitude of the eigenvalue variation may then be bounded as follows:

$$
\left|\delta \sigma_{l}\right| \leqslant \| \delta \boldsymbol{A}_{0}|| \underbrace{\left|\left(\hat{\boldsymbol{q}}_{l}^{+}, \boldsymbol{B} \cdot \hat{\boldsymbol{q}}_{l}\right)\right|^{-1}\left(\left|\hat{\boldsymbol{u}}_{l}\left(x_{0}, y_{0}\right) \| \hat{\boldsymbol{u}}_{l}^{+}\left(x_{0}, y_{0}\right)\right|\right)}_{S_{l}\left(x_{0}, y_{0}\right)}
$$

where $\left\|\delta \boldsymbol{A}_{0}\right\|$ is the norm of the structural perturbation. The quantity $S_{l}\left(x_{0}, y_{0}\right)$ defines the sensitivity of the eigenvalue $\sigma_{l}$ to a local structural perturbation $\delta \boldsymbol{A}_{0}$.

\section{REFERENCES}

Akervik, E., Hoepffner, J., Ehrenstein, U. \& Henningson, D. S. 2007 Optimal growth, model reduction and control in a separated boundary-layer flow using global modes. J. Fluid Mech. 579, 305-314.

Barkley, D., Gomes, M. G. M. \& Henderson, R. D. 2002 Three-dimensional instability in flow over a backward-facing step. J. Fluid Mech. 473, 167-190.

Butler, K. M. \& Farrell, B. F. 1992 Three-dimensional optimal perturbations in viscous shear flow. Phys. Fluids A 4, 1637-1650.

Chomaz, J. M. 2005 Global instabilities in spatially developping flows: non-normality and nonlinearity Annu. Rev. Fluid Mech. 37, 357-392.

Chomaz, J. M., Huerre, P. \& Redekopp, L. G. 1990 The effect of nonlinearity and forcing on global modes. In New Trends in Nonlinear Dynamcs and Pattern-Forming Phenomena (ed. P. Coulet \& P. Huerre), p. 259. Plenum.

Cossu, C. \& Chomaz, J. M. 1997 Global measures of local convective instabilities Phys. Rev. Lett. 78, 4387-4390.

Ding, Y. \& KawahaRA, M. 1999 Three-dimensional linear stability analysis of incompressible viscous flows using the finite element method Intl J. Numer. Meth. Fluids 31, 451-479.

Ehrenstein, U. \& Gallaire, F. 2005 On two-dimensional temporal modes in spatially evolving open flows: the flat-plate boundary layer. J. Fluid Mech. 536, 209-218.

Gallaire, F., Marquillie, M. \& Ehrenstein, U. 2007 Three-dimensional transverse instabilities in detached boundary layers. J. Fluid Mech. 571, 221-233.

Giannetti, F. \& Luchini, P. 2003 Receptivity of the circular cylinder's first instability. Proc. 5th Eur. Fluid Mech. Conf. Toulouse.

GiannetTi, F. \& Luchini, P. Structural sensitivity of the first instability of the cylinder wake. J. Fluid Mech. 581, 167-197. 
Griffith, M. D., Thompson, M. C., Leweke, T. , Hourigan, K. \& Anderson, W. P. 2007 Wake behaviour and instability of flow through a partially blocked channel. J. Fluid Mech. 582, 319-340.

Huerre, P. \& Monkewitz, P. A. 1990 Local and global instabilities in spatially developing flows Annu. Rev. Fluid Mech. 22, 473-537.

LANDAHL, M. T. 1980 A note on an algebraic instability of inviscid parallel shear flows. J. Fluid Mech. 98, 243-251.

Lehoucq, R. B., Masschhoff, K., Sorensen, D. \& Yang, C. 1996 ARPACK Software Package, http://www.caam.rice.edu/software/ARPACK/

LehoucQ, R. B., Sorensen, D. C. \& YANG, C. 1998 ARPACK Users's Guide. SIAM.

Marquet, O., Sipp, D., Chomaz, J.-M. \& Jacquin, L. 2008 Amplifier and resonator dynamics of a low-Reynolds number recirculation bubble in a global framework. J. Fluid Mech. 605, $429-443$.

Maslowe, S. A. \& Kelly, R. E. 1970 Finite-amplitude oscillations in a Kelvin-Helmholtz flow. Intl J. Non-Linear Mech. 5, 427-435.

Schmid, P. J. 2007 Nonmodal stability theory. Annu. Rev. Fluid Mech. 39, 129-162.

Schmid, P. J. \& Henningson, D. S. 2001 Stability and Transition in Shear Flows. Springer.

Schmid, P. J. \& Henningson, D. S. 2002 On the stability of a falling liquid curtain. J. Fluid Mech. 463, 163-171.

Theofilis, V. 2000 Globally unstable flows in open cavities. AIAA Paper 2000-1965.

Theofilis, V., Hein, S. \& Dallmann, U. 2000 On the origins of unsteadiness and threedimensionality in a laminar separation bubble. Phil. Trans. R. Soc. Lond. A 358, 3229-3246.

Trefethen, L. N., Trefethen, A. E., Reddy, S. C. \& Driscoll, T. A. 1993 Hydrodynamic stability without eigenvalues. Science 261, 578-584. 